\title{
Interpreting cues of goodness: thin sliced judgment of Malaysians and Chinese towards political leaders
}

\begin{abstract}
Thin slice studies have demonstrated that quick personality inferences of unknown others can be made fairly accurately. Utilizing focus group interviews, the current study examines thin sliced judgments and perceptions of six leaders in the context of goodness. Findings revealed that perceivers were able to form relatively accurate impressions of some leaders based on brief glimpses of nonverbal footage. Across all groups (Malaysian and Chinese) paralinguistic cues, dynamic cues and static cues were instrumental in the formation of positive perceptions of leaders in the context of goodness. These findings have implications for the areas of nonverbal behaviour, political communication, and personality judgement.
\end{abstract}

Keyword: Non verbal behavior/communication/cues; Thin slice; Person perception; Political leaders; Politicians; Goodness; Personality judgement; Collectivist cultures 